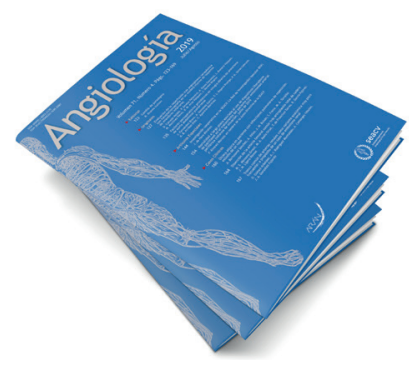

\title{
Original
}

\section{Factores perioperatorios pronósticos en cirugía abierta de aneurisma de aorta abdominal yuxtarrenal}

\section{Perioperative factors in open surgery of juxtarenal abdominal aortic aneurysm}

Jesús Fernández Bravo, Alejandro González García, Teresa Lidia Reyero Postigo, Patricia Lorena Torres Lebruno, Ana Begoña Arribas Díaz, César Aparicio Martínez

Servicio de Cirugía Vascular. Hospital Fundación Jiménez Díaz. Madrid

Palabras clave:

Aneurisma. Aorta. Yuxtarrenal.

Clampaje suprarenal Función renal.

\section{Resumen}

Introducción: a pesar del desarrollo de la técnica endovascular en el aneurisma de aorta abdominal, la reparación abierta sigue siendo considerada el gold-standard en los aneurismas de aorta abdominal yuxtarrenal.

Objetivo: evaluar los resultados del tratamiento quirúrgico del aneurisma de aorta yuxtarrenal, así como analizar los posibles factores que puedan influir en dichos resultados y la evolución que pueda presentar la función renal tras la cirugía.

Material y métodos: se realiza un análisis retrospectivo de los pacientes con aneurisma de aorta yuxtarrenal operados quirúrgicamente de manera programada desde 2010 hasta 2016. Se analizan factores de riesgo cardiovascular, hemoglobina y creatinina en sangre preoperatoria, tiempo y zona de clampaje, uso de perfusión renal, tiempo de intervención, creatinina posoperatoria y a largo plazo y necesidad de transfusión. También se describen morbilidad y mortalidad a los 30 días y fracaso renal agudo, siguiendo los criterios RIFLE. Por último, se analiza la posible relación de los factores con la morbimortalidad mediante la prueba de Chi-cuadrado.

Resultados: tras el análisis de un total de 51 pacientes, se presenta una mortalidad del 5,8\% a los 30 días y fracaso renal agudo del 3,9\%. Se observa que una hemoglobina preoperatoria $<13 \mathrm{~g} / \mathrm{dl}$ y la necesidad de transfusión de más de siete concentrados de hematíes durante el ingreso están asociados con mayor mortalidad y con mayor riesgo de fracaso renal agudo $(p<0,05)$. A su vez, los pacientes con insuficiencia renal crónica y con un nivel de ácido láctico elevado en sangre a las 24 horas se asocian a mayor riesgo de mortalidad a los 30 días $(p<0,05)$.

Conclusión: el estado basal del paciente antes de la cirugía abierta de aorta influye en los resultados de la misma. Es importante la optimización del mismo para disminuir la aparición de complicaciones.

\section{Abstract}

Introduction: despite the development of the endovascular technique in the abdominal aortic aneurysm, open repair is still considered as the gold-standard in juxtarenal abdominal aortic aneurysms.

Objective: to evaluate the results of the surgical treatment of the juxtarenal aortic aneurysm in our center, as well as to analyze the possible factors that may influence such results and the evolution that renal function may have after surgery.

Material and methods: a retrospective analysis of patients with juxtarenal aortic aneurysm surgically operated from 2010 to 2016 was performed, analyzing cardiovascular risk factors, hemoglobin and creatinine in preoperative blood analytics, time and area of clamping, use of renal perfusion, time of intervention, postoperative and longterm creatinine in blood analytics and need for transfusion. Morbidity and mortality at 30 days, and acute renal failure are also described, following the RIFLE criteria. Finally, we analyzed the possible relationship of the factors with morbidity and mortality through the Chi-square test.
Key words:

Aneurysm

Aorta. Juxtarenal.

Suprarenal

clamping. Rena

function.
Recibido: 04/02/2019 • Aceptado: 09/06/2019

Fernández Bravo J, González García A, Reyero Postigo TL, Torres Lebruno PL, Arribas Díaz AB, Aparicio Martínez C. Factores perioperatorios pronósticos en cirugía abierta de aneurisma de aorta abdominal yuxtarrenal. Angiología 2019;71(4):135-143.

DOl: http://dx.doi.org/10.20960/angiologia.00027

\section{Correspondencia:}

Jesús Fernández Bravo. Servicio de Cirugía Vascular. Hospital Fundación Jiménez Díaz. Avda. de los Reyes Católicos, 2. 28040 Madrid e-mail: jesusfkb@gmail.com 
Results: after the analysis of a total of 51 patients, we presented 30 days mortality of $5.8 \%$ and acute renal failure in 3.9\%. It is observed that a preoperative hemoglobin $<13 \mathrm{~g} / \mathrm{dl}$ and the need for transfusion of more than seven red cell concentrates during admission are associated with higher mortality at 30 days and with higher risk of acute renal failure $(p<0.05)$. Also, chronic renal insufficiency and a high level of lactic acid in patients' blood at 24 hours are associated with a greater risk of mortality at 30 days $(p<0.05)$.

Conclusion: the baseline status of the patient before open aortic surgery influences the results of the same. It is important to optimize it to reduce the appearance of complications.

\section{INTRODUCCIÓN}

Desde que se introdujo en 1991 el tratamiento endovascular para la reparación del aneurisma de aorta abdominal, se ha producido un mayor número de intervenciones mediante esta técnica, siendo en la actualidad usada hasta en el 70\% de las intervenciones del aneurisma de aorta abdominal (1).

Sin embargo, hasta un 15\% de los aneurismas de aorta abdominal son aneurismas yuxtarrenales, que por definición no presentan un cuello infrarrenal de suficiente longitud para permitir el clamplaje infrarrenal o el soporte de una endoprótesis (2).

A pesar del desarrollo de la técnica endovascular con la utilización de endoprótesis fenestradas o uso de chimeneas, muchos estudios siguen considerando la cirugía aórtica el gold-standard para los aneurismas yuxtarrenales (2-4).

En este estudio se analizan de forma retrospectiva los resultados en nuestro centro de la reparación mediante cirugía abierta del aneurisma de aorta yuxtarrenal.

El objetivo principal de este estudio es evaluar los resultados del tratamiento quirúrgico programado del aneurisma de aorta yuxtarrenal en nuestro centro.

Como objetivos secundarios se analizan los posibles factores que puedan influir en dichos resultados y la evolución a corto y largo plazo que pueda presentar la función renal tras la cirugía.

\section{MÉTODOS}

\section{Población a estudio}

Se realizó en nuestro centro un análisis retrospectivo de pacientes con aneurisma de aorta abdominal yuxtarrenal tratados de manera programada mediante cirugía abierta desde el 1 de enero de 2010 hasta el 31 de diciembre de 2016, ambos incluidos. El diagnós- tico de aneurisma yuxtarrenal se realizaba mediante tomografía axial computarizada (TAC) abdominal que presentaban todos los pacientes. Fueron considerados como tales aquellos aneurismas que no afectaban a las arterias renales pero no presentaban un cuello suficiente para el clampaje infrarrenal.

Se excluyeron los pacientes en los que, habiendo sido catalogados como aneurisma de aorta yuxtarrenal, durante el acto quirúrgico fue suficiente con la realización de un clampaje infrarrenal para su reparación.

\section{Variables preoperatorias analizadas}

Se recogieron en ambos grupos a través de la historia clínica las siguientes variables: edad, sexo, factores de riesgo cardiovascular (hipertensión arterial [HTA], diabetes mellitus [DM], dislipemia [DL], tabaquismo, insuficiencia renal crónica [IRC], cardiopatía isquémica [Cl], enfermedad pulmonar obstructiva crónica $[E P O C])$, paciente en diálisis, hemoglobina preoperatoria, hematocrito preoperatorio y creatinina en sangre preoperatoria.

A través del angio-TC se recogió y midió el diámetro del aneurisma.

Además, para la variable edad se realizó una nueva categoría separando los pacientes con 80 años o más para facilitar el análisis que se realizó posteriormente y su correcta interpretación. También se realizó una nueva categoría para la variable hemoglobina preoperatoria, considerando como anemia a pacientes con hemoglobina inferior a 13 mg/dl (este valor fue escogido ya que es el mínimo normal para varones, siendo el aneurisma de aorta abdominal más frecuente en ellos).

La decisión de realizar la reparación quirúrgica se decidía en sesión clínica en función de diámetro (> 55 varones y > 50 mujeres), síntomas y características del paciente. 


\section{Variables intraoperatorias y hospitalarias analizadas}

La intervención se realizaba mediante acceso retroperitoneal en la mayoría de los casos, con clampaje en función de la disponibilidad de cuello, valorado previamente mediante angio-TC e intraoperatoriamente de nuevo. Se utilizaba, en función del cirujano que realizaba la intervención, una perfusión de protección renal compuesta por suero Ringer frío de $500 c c+90$ cc de manitol + 5000 Ul heparina al $1 \%+250 \mathrm{mg}$ de actocortina. Durante la cirugía se utilizaba un recuperador de sangre.

Intraoperatoriamente se registraron tiempo de clampaje suprarrenal y lugar de clampaje, tiempo total de intervención, uso o no de suero de protección renal y sangrado aspirado intraoperatoriamente.

Se realizaron nuevas categorías para las variables tiempo de clampaje suprarrenal (tiempo largo para > 30 minutos), sangrado aspirado intraoperatoriamente (considerándose elevado $>1.500 \mathrm{ml}$ ) y tiempo total de intervención (considerándose elevado > 300 minutos) para poder facilitar el análisis posterior.

Se analizaron la estancia hospitalaria y la estancia en unidad de cuidados intermedios.

También se analizaron el ácido láctico, la hemoglobina, el hematocrito y la creatinina en sangre a las 24 horas de la cirugía.

Se analizó el número de concentrados de hematíes recibidos por el paciente durante la cirugía y posteriormente durante el ingreso.

El criterio de transfusión tras la cirugía fue de una hemoglobina $<9 \mathrm{mg} / \mathrm{dl}$ o $<10 \mathrm{mg} / \mathrm{dl}$ en pacientes con cardiopatía conocida.

Se realizaron nuevas categorías para las variables estancia hospitalaria (prolongada > 10 días), estancia en unidad de cuidados intermedios (prolongada $>48$ horas), número de concentrados de hematíes recibidos (elevados $>7$ concentrados) y ácido láctico (elevado si > $25 \mathrm{mg} / \mathrm{dl}$, al ser este el valor máximo normal).

\section{Seguimiento}

El seguimiento en consultas se realizó al mes, a los tres, seis y 12 meses y después del primer año, cada 12 meses mediante anamnesis, exploración física, control eco-doppler y, en caso necesario, angio-TC.

Se recogieron las complicaciones y los eventos adversos aparecidos a los 30 días posoperatorios, así como la supervivencia a largo plazo.

Se definieron como complicaciones mayores las complicaciones cardiacas, respiratorias, digestivas y renales y las reintervenciones. El resto de complicaciones fueron definidas como complicaciones menores.

También se recogió la creatinina en sangre al mes, a los tres meses, a los seis meses y al año de la cirugía.

\section{Definiciones}

Para la definición de insuficiencia renal crónica y de fracaso renal agudo durante el ingreso se siguieron los criterios RIFLE (5).

De este modo, se definió insuficiencia renal crónica en los pacientes que presentaban en la analítica preoperatoria una creatinina en sangre igual o mayor a $1,5 \mathrm{mg} / \mathrm{dl}$.

De igual manera, se definió para pacientes sin insuficiencia renal aguda la aparición de fracaso renal agudo cuando durante el ingreso presentaran una creatinina en sangre $>2 \mathrm{mg} / \mathrm{dl}$. Por otro lado, para los pacientes con insuficiencia renal crónica, el fracaso renal agudo se definía si presentaban durante el ingreso una duplicación de su creatinina basal.

Se definió como creatinina en sangre al alta a la última creatinina presentada por el paciente durante su ingreso.

Se definió como creatinina en sangre a largo plazo a la creatinina que se tenía del paciente a $>6$ meses de la cirugía.

\section{Análisis estadístico}

Se utilizó el programa estadístico IBM SPSS Statistics versión 23 para el cálculo estadístico del estudio.

Se realizó un análisis descriptivo de la muestra, realizando un análisis de la media, mediana y desviación típica para las variables edad, diámetro del aneurisma, creatinina en sangre preoperatoria, hemoglobina preoperatoria, tiempo de clampaje suprarrenal, 
sangrado aspirado intraoperatoriamente, ácido láctico a las 24 horas de la cirugía, estancia hospitalaria, número de concentrados de hematíes recibidos y creatinina en sangre al alta. Para el resto de variables descritas anteriormente, se calcularon la frecuencia y la frecuencia relativa.

Posteriormente, se realizó una comparación entre la mortalidad y morbilidad al mes y la aparición de fracaso renal agudo, con el resto de variables cualitativas descritas, para ver cuáles podían estar asociadas con la morbimortalidad y el deterioro renal. Para evaluar esta posible asociación se realizó la prueba de Chi-cuadrado o la prueba exacta de Fisher en función de la muestra.

También se analizó la evolución de la función renal mediante el análisis del cambio de la creatinina en cada paciente con la prueba de t de Student para muestras apareadas.

Por último, se realizó un análisis de Kaplan-Meier para valoración de supervivencia a largo plazo.

Se establece un error alfa de 0,05, aceptando un nivel de significación para $p<0,05$ en todos los test aplicados.

Los análisis fueron realizados según intención de tratar, teniendo por tanto en cuenta la totalidad de los pacientes.

\section{RESULTADOS}

\section{Población}

Desde enero de 2010 hasta diciembre de 2016 se operó a un total de 51 pacientes de aneurisma de aorta abdominal yuxtarrenal mediante cirugía abierta programada.

De estos, hubo 50 varones y una mujer. La edad media de estos pacientes fue de 72 \pm 7,6 años.

La media de seguimiento fue de 44,92 \pm 29,5 meses, con un seguimiento mayor de 60 meses en el 31,4\% de los pacientes.

Los distintos factores de riesgo presentados en estos pacientes se detallan en la tabla I.

El diámetro medio del aneurisma era de $64 \pm 16,5$ $\mathrm{mm}$, la hemoglobina preoperatoria media era de 14,2 $\pm 1,6 \mathrm{mg} / \mathrm{dl}$, el hematocrito preoperatorio medio era de $42,5 \pm 4,6 \%$ y la creatinina en sangre preoperatoria media, de 1,09 $\pm 0,35 \mathrm{mg} / \mathrm{dl}$.

\section{Cirugía}

El tiempo medio de la intervención fue de $306 \pm$ 48 minutos, siendo mayor de 300 minutos en 25 de los pacientes (49\%).

En 42 de los pacientes (82,3\%) se realizó un clampaje suprarrenal; en seis $(11,8 \%)$, uno supramesentérico; y en tres pacientes (5,9\%), uno supraceliaco.

El tiempo de clampaje suprarrenal medio fue de $25 \pm 7,3$ minutos y se superaron los 30 minutos en diez pacientes (19,6\%). No se superaron en ningún caso los 40 minutos.

La perfusión de protección renal se utilizó en 22 pacientes $(43,1 \%)$.

La cantidad media de sangre aspirada intraoperatoriamente fue de $1.166 \pm 627 \mathrm{ml}$, superando los $1500 \mathrm{ml}$ en nueve pacientes (17,6\%). Se obtuvo una recuperación media de sangre de $394 \pm 253 \mathrm{ml}$.

El número de concentrados de hematíes que recibieron los pacientes durante el ingreso presentó una media de 3,76 \pm 4,2 concentrados de hematíes, superando los siete concentrados de hematíes en siete pacientes (13,7\%). Doce de los pacientes (23,5\%) no precisaron de transfusión de hematíes durante el ingreso.

La hemoglobina posoperatoria media a las 24 horas era de 11,1 $\pm 1,26 \mathrm{mg} / \mathrm{dl}$, el hematocrito posoperatorio medio a las 24 horas era del 33,01 $\pm 3,9 \%$ y la creatinina posoperatoria a las 24 horas era de 1,16 $\pm 0,5 \mathrm{mg} / \mathrm{dl}$.

Por último, el ácido láctico en sangre a las 24 horas de la cirugía presentaba una media de 13,76 \pm 7,5, siendo elevado en seis pacientes (11,8\%).

\section{Estancia hospitalaria}

Los pacientes permanecieron hospitalizados una media de $10 \pm 10,7$ días. La mediana de hospitalización fue de ocho días. Presentaron una hospitalización prolongada (> 10 días) 15 de los pacientes (29,4\%).

La estancia media en UVI fue de 1,86 \pm 1,26 días. El 78,5\% de los pacientes precisaron de una estancia en la unidad de cuidados intermedios de 24-48 horas (29 pacientes 24 horas y once pacientes 48 horas), mientras que once de los pacientes $(21,5 \%)$ precisaron una estancia prologada (> 48 horas). 
Tabla I. Características de la población

\begin{tabular}{|l|c|}
\hline \multicolumn{1}{|c|}{ Variables } & $\begin{array}{c}\text { Aneurisma aorta yuxtarrenal } \\
\text { reparado mediante cirugía } \\
\text { abierta programada ( } \mathbf{n}=51)\end{array}$ \\
\hline Edad & 72 años $\pm 7,6$ \\
\hline Edad > 80 años & $11(21,6 \%)$ \\
\hline $\begin{array}{l}\text { Hipertensión } \\
\text { arterial }\end{array}$ & $30(58,8 \%)$ \\
\hline Diabetes mellitus & $10(19,6 \%)$ \\
\hline Dislipemia & $31(60,8 \%)$ \\
\hline Tabaco & $44(86,2 \%)$ \\
\hline $\begin{array}{l}\text { Insuficiencia } \\
\text { renal }\end{array}$ & $7(13,7 \%)$ \\
\hline $\begin{array}{l}\text { Cardiopatía } \\
\text { isquémica }\end{array}$ & $18(35,3 \%)$ \\
\hline $\begin{array}{l}\text { Enfermedad } \\
\text { pulmonar }\end{array}$ & $14(27,5 \%)$ \\
\hline $\begin{array}{l}\text { Hemoglobina } \\
\text { preoperatoria } \\
<13 \text { mg/dl }\end{array}$ & $10(19,6 \%)$ \\
\hline
\end{tabular}

\section{Seguimiento y complicaciones}

Durante el posoperatorio, fallecieron tres pacientes (5,9\% de mortalidad a 30 días) y diez presentaron complicaciones mayores (19,6\%), que fueron: dos, infarto de miocardio (3,9\%); dos, neumonía $(3,9 \%)$; dos, reintervenciones por shock hemorrágico-hipovolémico (3,9\%); dos, colitis isquémica $(3,9 \%)$; y dos, deterioro renal agudo (3,9\%). Un único paciente precisó terapia renal sustitutiva durante el posoperatorio $(1,9 \%)$ y falleció finalmente en el posoperatorio.

En cuanto a supervivencia a largo plazo, al año presentamos una supervivencia global del 92,2\%, que fue del 80,4\% a los cinco años (Fig. 1).

Durante el posoperatorio, los fallecimientos que tuvieron lugar fueron por las causas que se describen a continuación. El primero de ellos era un paciente de 65 años, EPOC severo, que presentó una intubación prolongada e insuficiencia respiratoria y falleció tras cuatro días en UVI. El segundo de ellos era un paciente de 75 años, cardiópata, que presentó ictus de probable origen cardioembólico con broncoaspiración posterior, que produjo su fallecimiento.

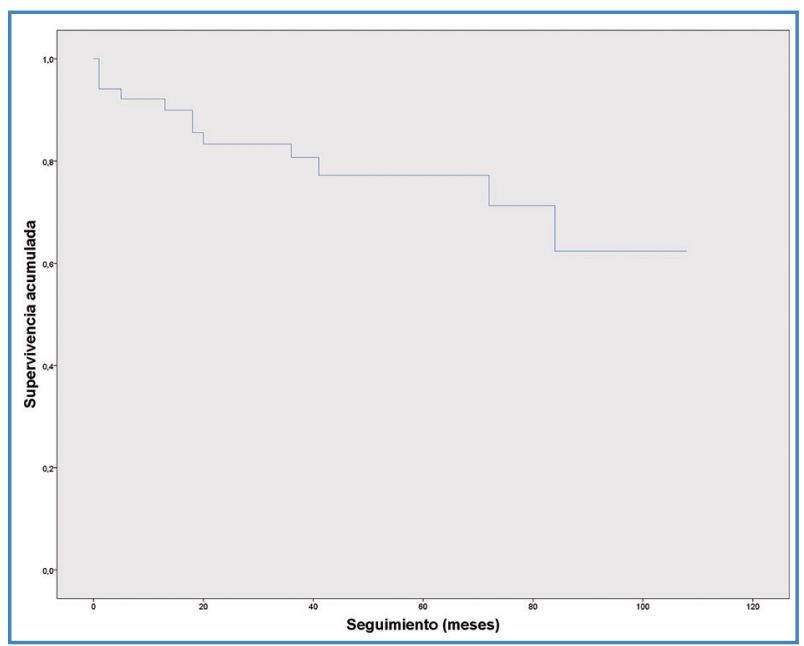

Figura 1. Análisis de supervivencia global Kaplan-Meyer de los pacientes con aneurisma de aorta abdominal yuxtarrenal operados mediante cirugía abierta programada, mostrándose supervivencia total en función del tiempo (meses).

El último de ellos era un paciente de 62 años, EPOC y con insuficiencia renal crónica que presentó en el posoperatorio un empeoramiento renal progresivo con repercusión neurológica (encefalopatía urémica), por lo que se le realizó terapia de reemplazo renal. En el posoperatorio presentó signos de isquemia intestinal con perforación y peritonitis, por lo que fue reintervenido, y finalmente falleció por shock séptico y fracaso multiorgánico.

\section{Evolución de la función renal}

Analizando la alteración de la creatinina en sangre de estos pacientes, observamos:

- La creatinina en sangre preoperatoria media era de 1,09 $\pm 0,35 \mathrm{mg} / \mathrm{dl}$.

- La creatinina en sangre al alta media fue de 1,06 $\pm 0,71 \mathrm{mg} / \mathrm{dl}$.

- La creatinina en sangre a largo plazo media fue de 1,16 $\pm 0,53 \mathrm{mg} / \mathrm{dl}$.

Tras la realización de un análisis mediante t de Student para muestras apareadas, se analizó cómo evolucionaba la creatinina en sangre previa a la cirugía, al alta y a largo plazo en cada paciente. Se observó un aumento de 0,18 mg/dl (0,05-0,30) de la creatinina en sangre a largo plazo con respecto a la creatinina en sangre al alta, siendo esta diferencia estadísticamente significativa $(p=0,05)$. 


\section{Impacto de los factores de riesgo}

Se realizó la prueba de Chi-cuadrado/prueba exacta de Fisher para evaluar la posible asociación entre las variables cuantitativas con la morbimortalidad y el deterioro renal, y se obtuvieron los siguientes resultados:

- Los pacientes con insuficiencia renal crónica presentaban una asociación positiva con respecto a mortalidad en comparación a aquellos con función renal normal: 28,6\% frente al 2,3\% $(p=0,046)$.

- Los pacientes con hemoglobina inferior a 13 $\mathrm{mg} / \mathrm{dl}$ previamente a la cirugía presentaban una asociación positiva con respecto a la mortalidad en comparación a aquellos con hemoglobina > $13 \mathrm{mg} / \mathrm{dl}$ : 30\% frente al $0 \%(p=0,001)$. También presentaban una asociación positiva con respecto a la incidencia de fracaso renal agudo: $20 \%$ frente al $0 \%(p=0,014)$.

- Los pacientes que precisaban de transfusión de $>7$ concentrados de hematíes durante el ingreso presentaban una asociación positiva con respecto a la mortalidad en comparación a aquellos con menor número de transfusiones: $28,6 \%$ frente al 2,3\% ( $p=0,046)$. También presentaban una asociación positiva con respecto a la incidencia de fracaso renal agudo: $28,6 \%$ frente al $0 \%(p=0,016)$.

- Los pacientes con ácido láctico en sangre elevado a las 24 horas presentaban una asociación positiva con respecto a la mortalidad en comparación a aquellos con ácido láctico en sangre normal a las 24 horas: 33,3\% frente al $4 \%(p=0,033)$.

- Los pacientes con cardiopatía isquémica previa u octogenarios no presentaron asociación con respecto a la mortalidad ( $p=1)$, así como tampoco la zona de clampaje $(p=0,692)$.

- La insuficiencia renal previa, el clampaje suprarrenal > 30 minutos y el uso de perfusión de protección renal no presentaban asociación con respecto a la incidencia de fracaso renal agudo ( $p=1)$, así como tampoco la zona de clampaje $(p=0,813)$.

- El resto de factores analizados (sexo, HTA, DM, $\mathrm{DL}$, tabaquismo, EPOC, tiempo total de interven- ción, sangrado aspirado intraoperatoriamente) no presentaron asociación con la incidencia de mortalidad o fracaso renal agudo $(p>0,05)$.

\section{DISCUSIÓN}

Con el desarrollo de la tecnología, ha habido un cambio en cuanto al tratamiento de los aneurismas de aorta abdominal, siendo más frecuente la reparación endovascular. Aproximadamente el $60 \%$ de los AAA infrarrenales son candidatos para la corrección mediante técnicas endovasculares y cabe esperar que este número aumente con la aparición de mejores endoprótesis (6). Esto conlleva que se realice el tratamiento quirúrgico en aquellos casos más complejos en donde la colocación de una endoprótesis no es factible, como los aneurismas yuxtarrenales y suprarrenales o explantes tras el fracaso de la endoprótesis. Estas intervenciones requieren el clampaje de la aorta a nivel suprarrenal o supravisceral.

Así, se observa en la literatura un mayor número de aneurismas tratados con clampaje suprarrenal (de un 6\% previo al primer EVAR a un 60\% en la actualidad) $(3,7)$.

El primer estudio publicado de aneurisma de aorta yuxtarrenal fue en 1986 por el grupo de Crawford y cols. (8), quienes observaron una mortalidad del $7,9 \%$ y una tasa de diálisis del $8 \%$. Una de las series más largas de la literatura, con 257 pacientes con aneurismas pararrenales y yuxtarrenales, publicada en 1999 por Jean-Claude y cols., presentaba una mortalidad del 5,8\% con una morbilidad renal del $41 \%$ y una tasa de hemodiálisis del 4,3\% (9). En 2002, Sarac y cols. publicaron los datos de la Clínica Cleveland, con 138 pacientes operados de aneurisma de aorta yuxtarrenal y una mortalidad del 5,1\%, una incidencia de fracaso renal agudo del $28 \%$ y una tasa de diálisis del 5,8\% (10). En 2008, West y cols., de la clínica Mayo, publicaron su experiencia con 247 pacientes, con una mortalidad del 2,7\%, una insuficiencia renal transitoria del $22 \%$ y necesidad de diálisis en el 3,7\% de los pacientes (11). Jongkind y cols. publicaron un metaanálisis en el que analizan los resultados de la cirugía del aneurisma yuxtarrenal en 21 estudios de 1986 a 2008, con un total de 1.256 pacientes. La mortalidad perioperatoria fue del 2,9\%, con una tasa 
de diálisis del 3,3\% (2). Por último, un metaanálisis más reciente es el realizado por Rao y cols. en 2015 (12), donde analizaron un total de 2.326 pacientes sometidos a cirugía abierta o reparación endovascular mediante fenestraciones al analizar 35 estudios de 1947 a 2013. Mostraban una mortalidad perioperatoria del 4,1\% tanto en reparación abierta como en endovascular, una incidencia de insuficiencia renal del 13,9\% en abierto frente al 11,4\% en endovascular, y necesidad de diálisis permanente en el 2,8\% de los pacientes con reparación abierta frente al 1,9\% en aquellos con endovascular.

Los resultados obtenidos en nuestro centro, con 51 pacientes, son similares a los publicados. Presentamos tasa de mortalidad perioperatoria ligeramente elevada con respeto a los estudios más recientes $(5,9 \%$ frente a 4,1\%) $(8-10,12)$. El hecho de presentar una población envejecida (el 21,6\% de la población eran octogenarios) podría estar relacionado con la mortalidad hallada dadas las comorbilidades que presentan habitualmente este tipo de pacientes. Además, en nuestra serie no ha habido pacientes que precisaran de diálisis y ha habido una incidencia de fracaso renal agudo del 3,9\% en comparación con otros estudios cuyas tasas eran superiores (2,8-12).

No todos los pacientes con aneurisma de aorta yuxtarrenal son candidatos a un clampaje suprarrenal. Si existe trombo o calcificación a nivel de aorta pararrenal o existe proximidad con la arteria mesentérica superior, podría ser necesario un control más proximal, por encima de las arterias viscerales, con el riesgo inherente de aumento de la poscarga cardiaca, hipotensión tras liberación o de provocar isquemia renal, hepática e intestinal o embolización distal $(9,13)$.

El lugar del control aórtico (supracelíaco frente a suprarrenal) ha sido motivo de debate en la literatura con respecto a la morbimortalidad posoperatoria.

Algunos autores como Green y cols. (14), que comparaban los resultados entre el clampaje supracelíaco y suprarrenal, mostraban tasas de mortalidad operatoria mayores en el clampaje suprarrenal (32\% frente a $3 \%)$ y mayor tasa de insuficiencia renal (23\% frente a 3\%). Atribuyeron estos resultados a la mayor probabilidad de desprender restos de placa aterosclerótica que en la aorta supracelíaca no es tan frecuente. Otros autores apuestan por la localización suprarrenal para el clampaje. En una revisión retrospectiva realizada por Sarac y cols. (10), compararon el clampaje supracelíaco frente al suprarrenal en los casos de aneurismas yuxtarrenales: la tasa de mortalidad fue mayor en el grupo supracelíaco (11,6 frente a 2,1\%), además de presentar una mayor tasa de insuficiencia renal (41,9 frente a 22,1\%).

En nuestro centro escogemos la mejor zona de clampaje previamente a la cirugía, con la evaluación de la aorta mediante el TAC preoperatorio. Preferimos siempre que sea posible el clampaje a nivel suprarrenal, seguido del supramesentérico y, por último, el supracelíaco. Para ello evaluamos la presencia de trombo intraluminal, ya que esto podría provocar la embolización del material trombótico en los vasos renales o viscerales con el clampaje. Además, en nuestro centro preferimos el acceso retroperitoneal para el tratamiento quirúrgico del aneurisma yuxtarrenal. Con él, se consigue una mayor visualización y control de la aorta a nivel de los vasos viscerales y evitar la posible sección de la vena, necesaria ocasionalmente con el acceso transperitoneal.

Como vemos en nuestros resultados, en el 82,3\% de los pacientes se realizó un clampaje suprarrenal, frente al 11,8\% de clampaje supramesentérico y el 5,9\% de clampaje supracelíaco. Además, tras el análisis realizado mediante Chi-cuadrado, no se vieron diferencias estadísticamente significativas con respecto a la mortalidad o el deterioro renal entre las distintas zonas de clampaje ( $p>0,05$ ).

La incidencia descrita de insuficiencia renal posoperatoria tras un control aórtico suprarrenal oscila entre el $14 \%$ y el $50 \%$ (15-17), lo que hace que sea la complicación posoperatoria más común y afecte negativamente a la supervivencia a largo plazo (21).

La etiología de la insuficiencia renal es multifactorial, pero existen varios factores que son constantes en la literatura. Estos factores son la presencia de insuficiencia renal preoperatoria $(10,16,17)$ y el tiempo de clampaje de la aorta suprarrenal (18-20).

Wahlberg y cols. (16) revisaron de manera retrospectiva 60 pacientes con aneurisma de aorta abdominal y clampaje suprarrenal temporal. Encontraron una concentración preoperatoria de creatinina de $1,21 \mathrm{mg} / \mathrm{dl}( \pm 0,54)$ y un tiempo de clampaje de 32 minutos ( \pm 17 minutos). Además, el 23\% de los pacientes presentaron una azotemia transitoria. 
La principal causa de la azotemia fue el tiempo de isquemia renal total, observándose un riesgo diez veces mayor con un tiempo de clampaje de la aorta suprarrenal $>50$ minutos.

En nuestro análisis no se observaron diferencias en cuanto a la mortalidad o el deterioro renal cuando el clampaje suprarrenal excedía los 30 minutos, si bien es cierto que en nuestra serie el tiempo máximo de clampaje suprarrenal fue de 40 minutos, sin que se superaran los 50 minutos descritos por Wahlberg y cols. (16).

Uno de los problemas fundamentales a la hora de analizar los resultados de la cirugía es la falta de consenso a la hora de definir el fracaso renal agudo en estos pacientes. Según Jongkind y cols. (2), se utiliza una definición distinta de fracaso renal agudo en cada estudio, por lo que el porcentaje de deterioro de la función renal posoperatoria varía ampliamente desde el 0 hasta el 39\%. Por ello, el propio Jongkind propone seguir los criterios diagnósticos de fracaso renal de la clasificación RIFLE publicada en 2004. En dicha clasificación se define el fracaso renal agudo según el filtrado glomerular/creatinina sérica y, por otro lado, según la diuresis. El deterioro del filtrado glomerular basal > 25\% es el criterio más sensible para detectar a aquellos pacientes en riesgo de desarrollar un fracaso renal agudo, mientras que un aumento al doble de la creatinina sérica basal es criterio de fracaso renal establecido (5). El grupo de Belkin publicó en 2009 un artículo en el que compararon los resultados de la cirugía aórtica con clampaje infrarrenal y con clampaje suprarrenal empleando unos criterios similares a los propuestos por el estudio RIFLE. Obtuvieron unos excelentes resultados, con un 7\% de deterioro de la función renal, un 0,6\% de hemodiálisis posoperatoria y una mortalidad menor del 2\% en el grupo con CSR (22).

En nuestro caso también hemos usado la clasificación RIFLE (5), ya que creemos que es esencial el consenso a la hora de definir los distintos conceptos que usamos en nuestra práctica diaria para poder comparar los resultados y mejorar nuestra labor.

En nuestro estudio, se observa un aumento estadísticamente significativo de $0,18 \mathrm{mg} / \mathrm{dl}(0,05-0,30)$ de la creatinina en sangre a largo plazo con respecto a la creatinina en sangre al alta $(p=0,05)$. Estos datos podrían indicarnos que el clampaje por encima de las renales no es tan inocuo y que tras la cirugía queda dañada la función renal. Sin embargo, hay que tomarlos con cautela, ya que el estudio no está dirigido al estudio de la función renal a largo plazo y este deterioro puede deberse al propio envejecimiento.

Por último, en nuestro estudio observamos un incremento significativo de la mortalidad en pacientes con insuficiencia renal crónica, anemia preoperatoria y politransfusión. Como ya hemos explicado anteriormente, el clampaje por encima de las arterias viscerales, necesario en la reparación abierta de estos aneurismas, puede provocar el empeoramiento renal del paciente, que, unido a un deterioro previo y un estado anémico, provoca mayor comorbilidad y mortalidad del paciente.

\section{Limitaciones}

Este estudio presenta las limitaciones propias de los estudios retrospectivos. También existe un sesgo de selección, ya que la anatomía aórtica de cada paciente determinaba varios de los factores analizados (zona de clampaje, tiempo quirúrgico, etc.). Además, se trata de un estudio de un único centro y con una muestra de estudio pequeña, por lo que puede que no se encuentren resultados significativos que puedan ser extrapolados a la población general.

\section{CONCLUSIONES}

Con nuestro estudio contribuimos a engrosar los datos que confirman la importancia de la optimización del paciente, previo a la intervención de un aneurisma aórtico.

Los pacientes que presentan una insuficiencia renal previa y/o anemia previamente a la cirugía abierta de aneurisma de aorta abdominal tienen una mayor mortalidad tras la cirugía. Igualmente, la necesidad de transfusión de concentrados de hematíes durante el ingreso es también un indicador de mala evolución.

La edad y un tiempo de clampaje suprarrenal mayor de 30 minutos pero inferior a 40 minutos no parecen influir por sí mismos en los resultados de la cirugía. 
El estado basal del paciente previamente a la cirugía abierta de aneurisma de aorta abdominal tiene influencia en los resultados de la propia cirugía. Es importante la optimización del paciente, mejorando en la medida de lo posible su estado basal previo a la cirugía para mejorar sus resultados, lo cual disminuye la aparición de complicaciones.

\section{BIBLIOGRAFÍA}

1. Brewster DC, Jones JE, Chung TK, Lamuraglia GM, Kwolek CJ, Watkins MT, et al. Long-term outcomes after endovascular abdominal aortic aneurysm repair: the first decade. Ann Surg 2006;244:426-38.

2. Jongkind V, Yeung KK, Akkersdijk G, Heidsieck D, Reitsma JB, Tangelder GJ, et al. Juxtarrenal aortic aneurysm repair. J Vasc Surg 2010;52:760-7.

3. Costin JA, Watson DR, Duff SB, Edmonson-Holt A, Shaffer L, Blossom GB. Evaluation of the complexity of open abdominal aneurysm repair in the era of endovascular stent-grafting. J Vasc Surg 2006;43:915-20.

4. Navarro TP, Bernardes RdC, Procopio RJ, Leite JO, Dardik A. Treatment of hostile proximal necks during endovascular aneurysm repair. Aorta 2014;2(1):28-36. DOl: 10.12945/j. aorta.2014.13-030.

5. Bellomo R, Ronco C, Kellum JA, Mehta RL, Palevsky P. Acute renal failure definition, outcome measures, animal models, fluid therapy and information technology needs: the Second International Consensus Conference of the Acute Dialysis Quality Initiative (ADQI) Group. Crit Care 2004;8:R204-12.

6. WolfYG, Fogarty TJ, Olcott C, Hill BB, Harris EJ, Mitchell RS, et al. Endovascular repair of abdominal aortic aneurysms: eligibility rate and impact on the rate of open repair. JVasc Surg 2000;32:519-23.

7. Landry G, Lau I, Liem T, Mitchell E, Moneta G. Open abdominal aortic aneurysm repair in the endovascular era: effect of clamp-site on outcomes. Arch Surg 2009;144:811-6.

8. Crawford ES, Beckett WC, Greer MS. Juxtarenal infrarenal abdominal aortic aneurysm. Special diagnostic and therapeutic considerations. Ann Surg 1986;203:661-70.

9. Jean-Claude JM, Reilly LM, Stoney RJ, Messina LM. Pararenal aortic aneurysms: the future of open aortic aneurysm repair. J Vasc Surg 1999;29:902-12.
10. Sarac TP, Clair DG, Hertzer NR, Greenberg RK, Krajewski LP, O'Hara PJ, et al. Contemporary results of juxtarenal aneurysm repair. J Vasc Surg 2002;36:1104-11.

11. West CA, Noel AA, Bower TC, Cherry KJ, Gloviczki P, Sullivan TM, et al. Factors affecting outcomes of open surgical repair of pararenal aortic aneurysms: a 10-year experience. J Vasc Surg 2006:43:921-7.

12. Rao R, Lane TR, Franklin IJ, Davies AH. Open repair versus fenestrated endovascular aneurysm repair of juxtarenal aneurysms. J Vasc Surg 2015;61:242-55.

13. Faggioli G, Stella A, Freyrie A, Gargiulo M, Tarantini S, Rodio $M$, et al. Early and long-term results in the surgical treatment of juxtarenal and pararenal aortic aneurysms. Eur J Vasc Endovasc Surg 1998;15:205-11.

14. Green RM, Ricotta JJ, Ouriel K, DeWeese JA. Results of supraceliac aortic clamping in the difficult elective resection of infrarenal abdominal aortic aneurysm. J Vasc Surg 1989:9:124-34.

15. El-Sabout RA, Reul GJ. Suprarenal or supraceliac aortic clamping during repair of infrarenal abdominal aortic aneurysms. Texas Heart Inst J 2001;28:254-64.

16. Wahlberg E, Dimurio PJ, Stoney RJ. Aortic clamping during elective operations for infrarenal disease: the influence of clamping time on renal function. J Vasc Surg 2002;36:13-8.

17. Sasaki T, Ohsawa S, Ogawa M, Mukaida M, Nakajima $T$, Komoda K, et al. Postoperative renal function after abdominal aortic aneurysm repair requiring a suprarenal aortic clamp. Surg Today 2000;30:33-6.

18. Desole A, Ferrari A, Tosato F, Milite D. Open repair for juxtarenal aortic aneurysm: short and long-term results. Ann Vasc Surg 2019;54:161-5. DOI: 10.1016/j.avsg.2018.05.071. E-pub Aug 6th, 2018.

19. Hoshina K, Nemoto M, Shigematsu K, Nishiyama A, Hosaka A, Miyahara T, et al. Effect of suprarenal aortic cross-clamping. Circ J 2014;78(9):2219-24. E-pub Jul 4th, 2014.

20. Yang SS, Park KM, Roh YN, Park YJ, Kim DI, Kim YW. Renal and abdominal visceral complications after open aortic surgery requiring supra-renal aortic cross clamping. J Korean Surg Soc 2012;83(3):162-70. DOI: 10.4174/ jkss.2012.83.3.162. E-pub Aug 27th, 2012.

21. Brewster DC, Cronenwett JL, Hallett JW, Johnston KW, KrupskiWC, Matsumura JS. Guidelines for the treatment of abdominal aortic aneurysm. J Vasc Surg 2003;37:1106-17.

22. Chong T, Nguyen L, Owens CD, Conte MS, Belkin M. Suprarenal aortic cross-clamp position: a reappraisal of its effects on outcomes for open abdominal aortic aneurysm repair. J Vasc Surg 2009;49:873-80. 